\title{
Diferencias individuales en dietantes crónicos. Influencia de los sistemas motivacionales en la alimentación
}

\author{
Jaime R. Silvala, Pablo Livacic-Rojas ${ }^{1,2 b}$, \\ Andrea Slachevsky C PhD ${ }^{3}$. \\ Differences among \\ chronic restrained eaters. \\ The influence of motivational systems
}

Background: Restrained eaters $(\mathrm{RE})$ are individuals who restrain their food intake on a regular basis as they are frightened to gain weight. However, they tend to overeat under conditions of anxiety. It has been shown that RE possess a behavioral inhibition system that is more active in tonic terms, which would partially explain their affective vulnerability. Even so, the influence of variations in the activation levels of the emotional systems on the eating behavior of a RE is still unknown. Our hypothesis is that variations of such systems will give place to two types of RE: a successful or a non-successful one. Aim: To assess the influence of variations on the activation of motivational systems in food intake of RE. Materials and methods: As part of a factorial experimental design, 105 undergraduate university students were part of an experimental test for inducting food intake. Then they reported their levels of dietary restraint and their emotional behavioral preferences. Results: Differences in the activation of motivational systems were significantly related to differences in food intake $(F=7.210 ; p=0.001)$. Additionally, food intake for those $\mathrm{RE}$ with a predominant inhibition system tended to be higher than for those with a more active approach system, though the latter did not reach a significant difference $(F=0.718 ; p=0.399)$. Conclusions: Although more investigations are required, our data suggest that the success of retaining the diet among the $\mathrm{RE}$ would depend on their profile of affective reactivity (affective style). There are putative implications for research on anorexia and obesity (Rev Méd Chile 2006; 134: 735-42).

(Key words: Anxiety; Anxiety disorders; Diet; Fasting)

\begin{abstract}
Recibido el 12 de abril, 2005. Aceptado el 22 de diciembre, 2005.
Trabajo financiado por el Fondo de Investigación y Desarrollo de la Universidad Mayor (2003) otorgado al primer autor.

${ }^{1}$ Laboratorio de Neurociencia Afectiva y Psicopatología, Facultad de Psicología, Universidad Mayor. ${ }^{2}$ Escuela de Psicología, Universidad de Santiago de Chile. ${ }^{3}$ Unidad de Neurología Cognitiva y Demencias, Servicio de Neurología, Hospital del Salvador, Facultad de Medicina, Universidad de Chile. Santiago de Chile.

aPsicólogo, candidato a doctor en Psicobiología.

bPsicólogo, Doctor en Psicología.
\end{abstract}

Correspondencia a: Jaime R. Silva. Laboratorio de Neurociencia Afectiva y Psicopatología, Facultad de Psicología, Universidad Mayor. Av. Alemania 0281, Temuco, IX región. Fax: (45) 206 313. E mail: jaimesilva@umayor.cl 
T os dietantes crónicos (DC) se conciben como $\perp$ individuos que limitan continuamente su alimentación por el miedo a subir de peso ${ }^{1}$. No obstante, numerosas investigaciones han demostrado que bajo ciertas condiciones los DC aumentan sus niveles de ingesta, sobrealimentándose ${ }^{2-10}$. Tales condiciones incluyen el consumo de una comida prohibida, la ingesta de alcohol o el experimentar estados emocionales intensos ${ }^{11}$. De las condiciones comunicadas ${ }^{11}$, el comportamiento emocional ha recibido un especial interés de parte de los investigadores, particularmente la ansiedad $^{12}$. En efecto, el consumo incrementado de los DC es especialmente pronunciado cuando una fuente de estrés amenaza a la identidad personal (i.e. falla en una tarea) en oposición a una amenaza física (i.e. choque eléctrico) ${ }^{3}$. Por ello, se ha propuesto que lo anterior es producto de una forma particular de autorregulación emocional $^{5}$.

Sin embargo, existen controversias y resultados disímiles entre los investigadores de la restricción alimenticia. Es una observación común que, en los procedimientos experimentales para ocasionar la sobreingesta en DC, una buena parte de los sujetos con altos niveles de restricción no desinhiben su alimentación ${ }^{13}$. Para algunos investigadores esto es producto de la existencia de dos tipos de dietantes, los Dietantes Exitosos y los Dietantes No Exitosos ${ }^{14}$. Aquéllos serían individuos que muestran una alta restricción alimenticia con una baja susceptibilidad a fracasar en ella y éstos los que combinan una alta restricción con una alta susceptibilidad a sobrealimentarse.

Sistemas motivacionales, asimetrías frontales y restricción alimenticia. Existe cierto nivel de acuerdo en la comunidad científica acerca de los principios generales que rigen a la respuesta emocional humana. Esta conceptualización se deriva de un enorme cúmulo de evidencia que apunta a la existencia de dos sistemas generales de motivación que organizarían y caracterizarían el comportamiento emocional ${ }^{15,16}$. Así, el procesamiento de la información emocional derivaría de dos sistemas ortogonales cuya operación se manifestaría en un repertorio conductual unidimensional ${ }^{17}$. Los sistemas motivacionales serían, el sistema motivacional de activación/aproximación (Behavioral Activation System [BAS]), cuya representación cortical se ha asociado a la corteza prefrontal izquierda, y el sistema motivacional de inhibición (Behavioral Inhibition System [BIS]), cuya representación cortical se ha asociado a la corteza prefrontal derecha ${ }^{18}$. Estos sistemas organizan la respuesta emocional de modo diferente; por un lado, el BAS dirige el comportamiento hacia metas y hacia estímulos reforzantes, siendo responsable de la emergencia de cierto tipo de afectos positivos; por el otro, el BIS organiza el comportamiento para evitar o huir de un evento/ estímulo aversivo, siendo responsable de la aparición de afectos negativos. En la funcionalidad de aquellos sistemas puede existir cierta asimetría en su nivel de actividad, de tal modo que alguno de ellos puede ser preponderante a la hora de organizar la conducta. Las asimetrías en los sistemas motivacionales se han relacionado a distintas formas de psicopatología ${ }^{16}$, una de las cuales ha sido el comportamiento alimentario anormal ${ }^{19}$. Puntualmente, Silva et al observaron que los DC presentan una asimetría en la actividad de la corteza prefrontal derecha, un patrón de actividad cerebral relacionado a respuestas ansiosas exacerbadas frente a la adversidad $15,20-23$ y a dificultades en la regulación emocional ${ }^{24}$.

Tal hallazgo es congruente con lo revelado por los estudios conductuales antes descritos, donde desinhibición alimenticia se ha asociado preferentemente al DC cuyo estado emocional predominante es la ansiedad. De esta forma puede entenderse que los DC se sobrealimentan debido a la intensidad o frecuencia en que experimentan esa emoción, lo cual, a su vez, estaría influido por un patrón específico de actividad cerebral (una asimetría en la actividad BIS/BAS, donde el BIS aparece como predominante). Sin embargo, no todo DC presentó este patrón de asimetría en la activación cerebral, mostrando que tales marcadores psicofisiológicos no son condición necesaria ni suficiente para producir» un DC. Aun así, estos datos sugieren que las diferencias en la actividad de los sistemas motivacionales podrían estar relacionadas al hecho ampliamente descrito de que no todo DC desinhibe su alimentación en condiciones que favorecen la ansiedad ${ }^{13}$. Lamentablemente, una de las limitaciones del estudio de Silva et $\mathrm{al}^{19}$ es la utilización de la Escala de Restricción sin la realización de una prueba de desinhibición alimenticia. Dado que existe una enorme variación inte- 
rindividual en el fenómeno de desinhibición, se hace necesario complementar dicho estudio con una investigación acerca de la relación entre los sistemas motivacionales y la desinhibición alimenticia como tal, y no solamente con el nivel de restricción reportado. Es por ello que planteamos la necesidad de investigar la influencia de disposiciones generales (niveles de asimetría de los sistemas motivacionales) en la conducta alimenticia de los DC, existiendo una continuidad conceptual entre la investigación de Silva et al (2002) y el presente estudio. En general, lo que se esperaría hallar es un patrón temperamental de vulnerabilidad a la ansiedad (un sistema motivacional de inhibición más activo) en dietantes poco exitosos (aquellos se sobrealimentan o desinhiben su alimentación) y un patrón opuesto (o al menos diferente) en los dietantes más exitosos (aquellos que persisten en su restricción). En este contexto, esperamos que el sistema BIS sea preponderante en aquellos dietantes que no son exitosos en restringir su alimentación, ya que al experimentar mayor frecuencia/ intensidad de ansiedad, estos sujetos deberían constantemente enmascarar dicho afecto dando lugar a la sobrealimentación. Por otro lado, en los dietantes exitosos debiéramos encontrar un sistema BAS más activo, ya que al experimentar menos ansiedad estos sujetos debieran recurrir menos al enmascaramiento y la sobrealimentación.

Por lo tanto, dados los antecedentes teóricos disponibles, el problema de investigación que nos guía puede formularse a través de la siguiente pregunta: en una situación experimental que induce la sobrealimentación existe relación entre el nivel de activación basal de los sistemas de motivación (de aproximación e inhibición), y las diferencias en el monto de consumo de los dietantes crónicos? El estado actual del campo de estudios de DC y de la respuesta emocional humana, nos permiten hipotetizar que existirán diferencias significativas en el consumo alimenticio, dependientes del nivel de asimetría de activación de los sistemas de motivación en los DC. Específicamente, la hipótesis general es que los dietantes más restrictivos tendrán un sistema de aproximación/activación conductual basalmente más activo en comparación con su contraparte menos restrictiva, mientras que el patrón inverso espera encontrarse para estos últimos.

\section{Material y MÉTOdo}

Participantes. El tipo de muestro utilizado fue probabilístico y la muestra obtenida se extrajo mediante una selección aleatoria simple. El total de participantes fue de 105 mujeres estudiantes de pregrado de cinco carreras distintas de la Universidad Mayor, pertenecientes a las ciudades de Temuco y Santiago (para descripción de la muestra ver Tabla 1). Cabe señalar que, del total de sujetos seleccionados, no se reportaron rechazos para participar en ningún caso y su participación fue voluntaria.

Metodología. Experimental.

Diseño. Factorial completamente aleatorizado (FCA-pqr). Este diseño se utilizó para evaluar las

Tabla 1. Estadísticos descriptivos de la muestra por variables de interés

\begin{tabular}{|lcccr|}
\hline & Desviación media & Estándar & Valor mínimo & Valor máximo \\
\hline Edad & 19,46 & 1,93 & 17 & 27 \\
Estatura & 159,79 & 5,40 & 146 & 172 \\
Peso corporal & 57,55 & 8,90 & 42 & 100 \\
Índice de masa corporal & 22,57 & 3,58 & 17,72 & 41,62 \\
Escala de restricción & 11,13 & 5,70 & 0 & 31 \\
Preocupación por la dieta & 6,70 & 3,62 & 0 & 18 \\
Asimetría BIS/BAS & 0 & 1,23 & $-2,85$ & 3,42 \\
& 105 & & & \\
$\mathrm{~N}$ & & & & \\
\hline
\end{tabular}


hipótesis referidas a los efectos de cada variable independiente por sí sola, así como los efectos de la combinación entre sus distintos niveles.

Instrumentos. Restricción alimenticia: Se utilizó la Escala de Restricción ${ }^{1}$, la cual evalúa actitudes hacia la alimentación, frecuencia de dietas y fluctuación de peso. Esta escala mostró propiedades psicométricas adecuadas en una población universitaria chilena (sometidas a publicación). Específicamente, el índice de fiabilidad de la prueba fue de aaa $=0,8104$. Respecto a la validez de constructo factorial, se realizó un análisis factorial confirmatorio, utilizando el método de extracción de factorialización de ejes principales con el método de rotación Equamax. Los resultados obtenidos a partir de los autovalores arrojan dos factores (autovalor 1=3.755; autovalor $2=1.393$ ), con un porcentaje de varianza explicada total de 40.284. Al coincidir los ítems con los factores obtenidos en la población diana, los puntajes obtenidos a través de la versión en español de la escala son susceptibles de interpretar en términos del constructo en cuestión.

Disposiciones anímicas: Se utilizó el «Cuestionario de Afecto Positivo y Negativo» (PANAS, Positive \& Negative Affect Schedule) ${ }^{25}$. Este cuestionario permite obtener una puntuación en afectividad positiva (subescala PA) y otra en afectividad negativa (subescala NA).

Sistemas de motivación: Se utilizó la Escala BIS/BAS (Behavioral Inhibition System \& Behavioral Activation System) ${ }^{26}$ para obtener un índice indirecto de las asimetrías de los sistemas cerebrales de motivación -para el procedimiento matemático ver Sutton y Davidson, 199718_. Se diferenció a los grupos en base a un corte en la mediana de los puntajes a fin de no subestimar la variabilidad de la muestra.

Procedimiento. La investigación se llevó a cabo siguiendo los lineamientos éticos internacionales y de la Universidad Mayor, mediante el procedimiento estándar para la provocación de sobrealimentación en los participantes ${ }^{1,13}$. Los sujetos acudieron individualmente al laboratorio, en donde firmaron un certificado de consentimiento para participar de la investigación y un cuestionario médico general. El estudio se implementó en tres fases: fase pre, fase experimental y fase post experimental, utilizando la técnica de doble ciego a fin de garantizar la ausencia de sesgos en la observación. A los participantes se les explicó que el experimento consistiría en evaluar el efecto de la temperatura en la percepción de sabor». Para tal efecto, fueron asignados al azar, mediante el uso de un listado de personas y una tabla de número aleatorios, a las tres condiciones experimentales. El paso anterior se implementó a fin de equilibrar el diseño experimental y con ello obtener mejores estimadores estadísticos, así como también, estandarizar las diferentes condiciones experimentales (〈control») un aperitivo» 0 〈dos aperitivos»). En la fase pre, los participantes del grupo control no recibieron ningún alimento. Durante esta misma fase, los participantes del grupo experimental an aperitivo» y «dos aperitivos», recibieron una porción de 300 cc y una de 600 cc de leche batida de chocolate, respectivamente. Posteriormente, en la fase de tratamiento se entregaron dos recipientes con helado, de 900 grs, solicitando mediante instrucciones la evaluación del sabor de cada helado a través de un cuestionario. Una vez concluido éste, se les dijo a los participantes que spodían tomar todo el helado que quisieran pues luego sería desechado»y se les dejó solos durante 10 min. Finalmente, se implementó la fase post tratamiento, donde los participantes respondieron los autorreportes y se les midió el peso y la estatura. Luego, se les explicó las verdaderas razones de la investigación y se les pidió que guardaran el secreto acerca del experimento.

\section{RESUlTADos}

Los resultados muestran que cuando se evaluaron los efectos de cada factor por sí sólo, no existió significación estadística. A su vez, bajo condiciones de interacción, sólo se encuentran efectos experimentalmente significativos cuando los factores grupo experimental, asimetría BIS/BAS y dietante crónico interactúan entre sí, $\mathrm{F}(2,102)=$ 7,$210 ; p=0,001$. Para esta condición, como para las restantes, se cumplen los supuestos de la prueba ANOVA (Normalidad de las puntuaciones $\mathrm{Z}$ de Kolmogorov-Smirnov $[0,968 ; p=0,306]$ y prueba de homogeneidad de varianzas de Levene con $F$ $[11,93]=0,808 ; p=0,632)$. Los efectos experimentales fueron captados con una alta sensibilidad 
(potencia $=0,927) \mathrm{y}$, teniendo un impacto bajo en los participantes del estudio (tamaño del efecto $=0,134$ ). Además, cuando se comparan las medias marginales de los dietantes a partir de puntajes de las medianas de asimetría, éstos no arrojan diferencias estadísticamente significativas $(\mathrm{F}=0,718 ; \mathrm{p}=0,399)$ (Tabla 2). Sin embargo, al observar la tendencia de las medias de consumo en cada condición, se verifica la dirección planteada por nuestras hipótesis aun cuando no alcanzan el nivel de significancia (Figura 1). Debido al tamaño del efecto encontrado, es posible hipotetizar que en una muestra de mayor tamaño con individuos con puntuaciones más extremas en la variable asimetría, estas diferencias se expresarían con un nivel de significancia estadística y empírica.

\section{DisCUSIÓN}

Los datos obtenidos son congruentes con la hipótesis planteada inicialmente. Efectivamente, las diferencias en los puntajes de asimetría de los sistemas motivacionales BIS/BAS, en el contexto de una prueba experimental de desinhibición de la alimentación, se relacionan con diferencias en el consumo alimenticio del DC. Específicamente, los DC con asimetrías BIS (DCBIS) tienden a consumir más alimento que el DC con asimetrías BAS (DCBAS), aunque este último dato no alcan- zó una diferencia estadísticamente significativa. Aun así, los hallazgos nos permiten proponer que, cuando se considera el fenómeno de la sobrealimentación en dietantes crónicos, las variaciones en las predisposiciones afectivas son una variable a considerar.

Así, podemos sugerir que las personas que realizan dietas crónicamente serían más vulnerables a la sobrealimentación según su perfil de reactividad afectiva (estilo afectivo). En efecto, los DC cuyo sistema de evitación es más activo tienden a sobrealimentarse mientras que los DC cuyo sistema de aproximación es más activo tienden a sostener su restricción.

Deben tenerse en cuenta algunas limitaciones de este estudio. En primer lugar, tanto las medidas de restricción y de sistemas motivacionales derivan de autorreportes, un método de investigación con limitaciones ampliamente reportadas. Por ello es necesario utilizar medidas psicofisiológicas a fin de verificar con mayor precisión la consistencia de los datos. En segundo lugar, aunque la potencia $(0,93)$ de los resultados asegura la detención de la interacción de las variables, el tamaño del efecto $(0,13)$ hace necesario replicar este estudio utilizando una muestra con grupos más numerosos y con puntuaciones más extremas en los autorreportes. Con ello se podría precisar el impacto de las variables motivacionales y de restricción alimenticia sobre el consumo de ali-

Tabla 2. Estadísticos descriptivos por factores y condición

\begin{tabular}{|lllcrr|}
\hline $\begin{array}{l}\text { Grupo } \\
\text { Experimental }\end{array}$ & & Asimetría & $\begin{array}{c}\text { Media de } \\
\text { consumo }\end{array}$ & $\begin{array}{c}\text { Desviación } \\
\text { estándar }\end{array}$ & N \\
\hline Sin aperitivo & No dietante & BIS & 136,00 & 38,82 & 5 \\
& & BAS & 59,44 & 44,82 & 9 \\
& Dietante & BIS & 103,85 & 53,74 & 13 \\
Aperitivo 1 & \multirow{2}{*}{ No dietante } & BAS & 135,00 & 83,28 & 8 \\
& & BIS & 105,56 & 77,72 & 9 \\
& Dietante & BAS & 91,11 & 40,76 & 9 \\
Aperitivo 2 & \multirow{2}{*}{ No dietante } & BIS & 123,75 & 75,92 & 12 \\
& & BAS & 128,00 & 68,70 & 5 \\
& Dietante & BIS & 56,57 & 51,23 & 14 \\
& & BIS & 125,50 & 97,82 & 10 \\
& & BAS & 160,00 & 87,37 & 4 \\
& & & 75,00 & 68,68 & 7 \\
\hline
\end{tabular}




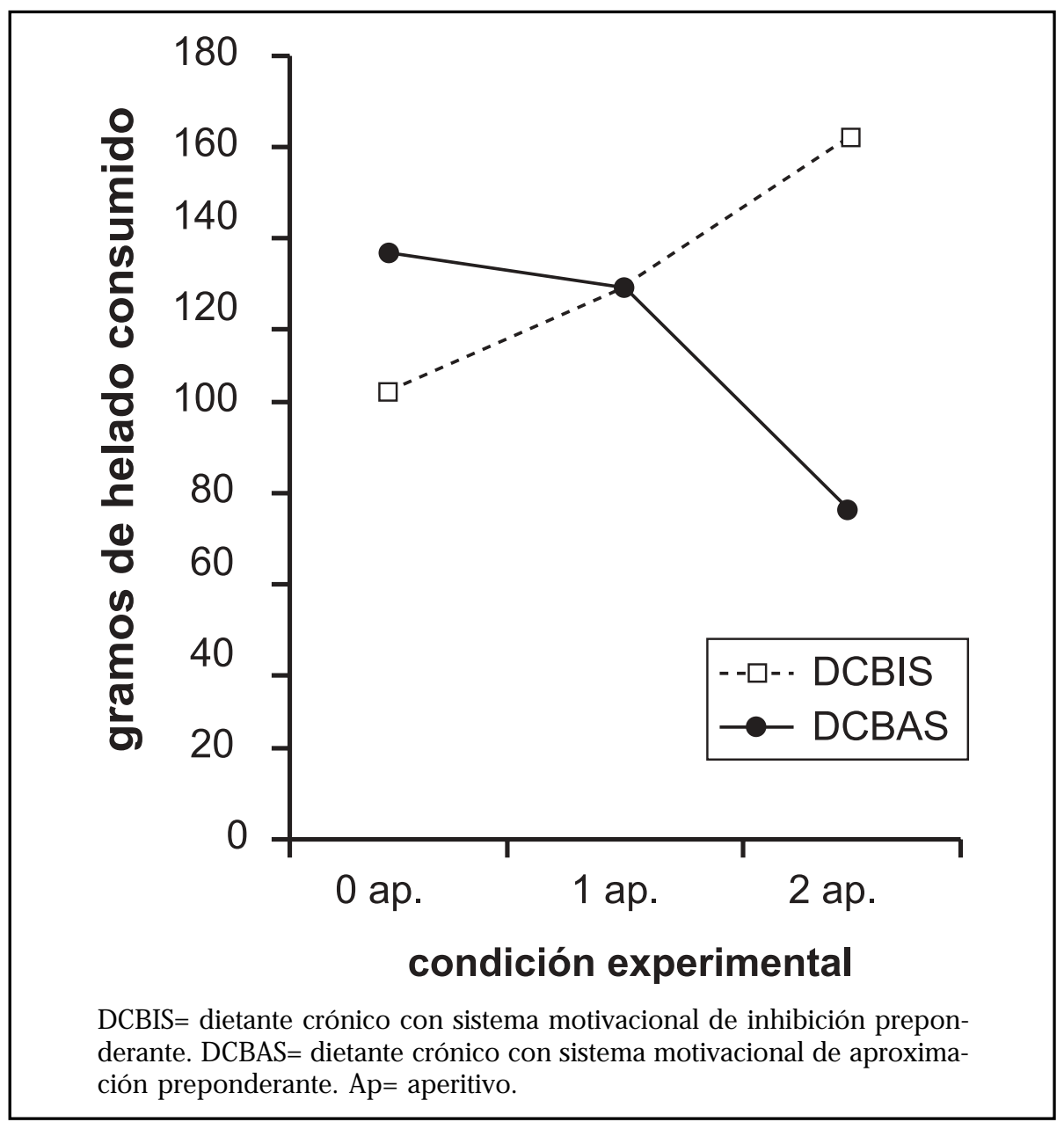

Figura 1. Consumo de helado en dietantes crónicos según tipo de asimetría del sistema motivacional.

mentos del DC, en situaciones que favorecen la desinhibición alimenticia.

La dirección futura de la presente conceptualización se enmarca dentro del estudio de los desórdenes alimentarios y la obesidad. Pudiendo identificar patrones distintivos confiables, en el estilo afectivo y el comportamiento alimentario de los DC, registrado a través de medidas biológicas (asimetrías frontales) y/o autorreportes (asimetrías BIS/BAS), nos encontramos en posición para la realización de estudios longitudinales, tendientes al esclarecimiento de parte de la etiología de ciertos trastornos alimentarios (i.e. anorexia) y la obesidad.
En efecto, considerando la asociación entre restricción alimenticia y el desarrollo de anorexia ${ }^{27}$ y obesidad $^{28}$ y mediante la diferenciación de patrones distintivos dentro de la población con riesgo a padecer dichas condiciones (dietantes crónicos), será posible establecer probables sendas de desarollo en esas alteraciones ${ }^{12}$. Específicamente, si las hipótesis planteadas son correctas, debiera esperarse que: (a) aquellos DC con un alto nivel de activación de BIS estén propensos a desarrollar obesidad, mientras que (b) aquellos DC con un alto nivel de activación de BAS sean propensos a desarrollar trastornos alimentarios del tipo anoréxi- 
co. Tal como se ha propuesto ${ }^{12}$, si un DCBIS es expuesto a múltiples situaciones que actualizan su vulnerabilidad a la ansiedad, es muy probable que en el largo plazo, la continua necesidad de enmascarar la ansiedad y la sobrealimentación correspondiente lleven al individuo a aumentar su peso corporal a niveles anormales. Del mismo modo, un sujeto DCBAS que esté sometido a múltiples situaciones que favorecen la ansiedad, en su continua mantención de metas como la dieta,

\section{REFERENCIAS}

1. Herman P, Polvy J. Restrained eating. En: Stunkard A, ed. Obesity. Philadelphia: Saunders 1980; 208-25.

2. BAuCon D, Aiken P. Effect on depressed mood on eating among obese and nonobese dieting and nondieting persons. J Pers Soc Psychol 1981; 41: 577-85.

3. Heatherton T, Baumeister R. Binge-eating as escape from self-awareness. Psychol Bull 1991; 126: 890-900.

4. Polvy J, Herman P. Dieting and binding: a causal analysis. Am Psychol 1985; 40: 193-201.

5. Polivy J, Herman P. Distress and eating: why do dieters overeat? Int J Eat Disord 1999; 26: 153-64.

6. Polivy J, Herman P. The effect of resolving to diet on restrained and unrestrained eaters: the «alse hope syndrome». Int J Eat Disord 1999; 26: 434-47.

7. Sheppard-Sawyer C, McNaliy R, Fischer J. Filminduced sadness as a trigger for disinhibited eating. Int J Eat Disord 2000; 28: 215-20.

8. TANo FSKY-KrafF M, WilfiEy D, SPURRELL E. Impact of interpersonal ego-related stress on restrained eaters. Int J Eat Disord 2000; 27: 411-8.

9. Stroud L, Tanofsky-KrafF M, Wilfiey D, Salovey P. The Yale interpersonal stressor (YIPS): affective, physiological, and behavioral responses to a novel interpersonal rejection paradigm. Ann Behav Med 2000; 22: 204-13.

10. Boon B, Stroebe W, Schut H, Ijntema R. Ironic processes in the eating behavior of restrained eaters. Br J Health Psychol 2002; 7: 1-10. luchará contra la tendencia a «enmascarar»aumentando la sensación positiva de sí mismo en la medida que mantienen su restricción (así a mayor ansiedad mayor restricción). En el largo plazo esto podría desencadenar un DC que inhibe su alimentación hasta niveles patológicos (i.e. anorexia). Hallazgos de este tipo tendrían consecuencias significativas en la conceptualización y las políticas respecto del tratamiento y prevención de desórdenes alimentarios y obesidad.

11. Ruderman A. Dietary restraint: a theoretical and empirical review. Psychol Bull 1986; 99: 247-62.

12. SiLva J. Ansiedad y sobrealimentación. Investig Cienc 2005; 341: 33-4.

13. Polvi J, Heatherton T, Herman P. Self-esteem, restraint, and eating behavior. J Abnorm Psychol 1988; 97: 354-6.

14. Van StRien T. The concurrent validity of a classification of dieters with low versus high susceptibility toward failure of restraint. Addict Behav 1997; 22: 587-97.

15. Davidson RJ. Emotion and affective style: hemispheric substrates. Psychol Sci 1992; 3: 3943.

16. SiLva J. Biología de la regulación emocional: su impacto en la psicología del afecto y la psicoterapia. Terapia Psicológica 2003; 21: 163-72.

17. Cacioppo J, Gardner W, Berntson G. The affect system has parallel and integrative processing components: form follows function. J Pers Soc Psychol 1999; 76: 839-55.

18. SuTton SK, Davidson RJ. Prefrontal brain asymmetry: a biological substrate of the behavioral approach and inhibition systems. Psychol Sci 1997; 8: 204-10.

19. Silva J, Pizzagaui D, Larson C, Jackson D, Davidson RJ. Frontal brain asymmetry in restrained eaters. J Abnorm Psychol 2002; 111: 676-81.

20. Davidson RJ. Affective style and affective disorders: perspectives from affective neuroscience. Cognition and Emotion 1998; 12: 307-30.

21. Davidson RJ, Irwin W. The functional neuroanatomy of emotion and affective style. Trends Cogn Sci 1999; 3: 11-21. 
22. Davidson RJ, Jackson D, KaLin N. Emotion, plasticity, context, and regulation: Perspectives from affective neuroscience. Psychol Bull 2000; 126: 890-909.

23. Buss KA, Malmstadt JR, Dolski I, Kalin NH, GoldsmTH HH, Davidson RJ. Right frontal brain activity, cortisol, and withdrawal behavior in 6-month-old infants. Behav Neurosci 2003; 117: 11-20.

24. Larson C, Sutton SK, Davidson RJ. Affective style, frontal EEG asymmetry and the time course of the emotion-modulated startle response. Psychophysiology 1998; 35: S52.
25. Watson D, Ciark LA, Tellegen A. Development and validation of brief measures of positive and negative affect: The PANAS scales. J Pers Soc Psychol 1988; 54: 1063-70.

26. Carver C, White T. Behavioral inhibition, behavioral activation, and affective responses to impending reward and punishment: the BIS/BAS scales. J Pers Soc Psychol 1994; 67: 319-33.

27. Polivy J, Herman P. Causes of eating disorders. Annu Rev Psychol 2002; 53: 187-213.

28. Јевв S. Aetiology of obesity. Br Med Bull 1997; 53: 264-85.

\section{Agradecimientos}

Los autores agradecen a Catherine Cerna, Jorge del Canto, Claudia Hernández, Kyzzy Mainhard y Ximena Peralta por su ayuda en la implementación de la situación experimental. Adicionalmente agradecemos a un revisor anónimo por sus valiosos comentarios y sugerencias. 